\title{
Questes
}

\section{Topiques de la saison inverse : hiver, désamour et pauvreté dans la littérature médiévale}

\section{Sarah Delale}

\section{(2) OpenEdition}

\section{Journals}

\section{Édition électronique}

URL : http://journals.openedition.org/questes/4373

DOI : 10.4000/questes.4373

ISSN : 2109-9472

\section{Éditeur}

Les Amis de Questes

\section{Édition imprimée}

Date de publication : 15 décembre 2016

Pagination : $33-53$

ISSN : 2102-7188

\section{Référence électronique}

Sarah Delale, «Topiques de la saison inverse : hiver, désamour et pauvreté dans la littérature

médiévale », Questes [En ligne], 34 | 2016, mis en ligne le 21 décembre 2016, consulté le 01 mai 2019. URL : http://journals.openedition.org/questes/4373; DOI : 10.4000/questes.4373 


\title{
Topiques de la saison inverse : hiver, désamour et pauvreté dans la littérature médiévale
}

\author{
Sarah DELALE \\ Université Paris-Sorbonne
}

«Yver, vous n'estes q'un villain !'», s'exclame Charles d'Orléans, assimilant la saison froide à l'un des personnages de la fiction médiévale qui s'oppose le plus aux codes littéraires ${ }^{2}$. Les textes médiévaux ont souvent réinvesti la pensée binaire des saisons en liant intimement l'été « plaisant et gentil ${ }^{3}$ » à la littérature, tandis que l'hiver renvoyait à l'obscurité et au silence. L'ouverture printanière, qui caractérise la plupart des textes des XII et XIII ${ }^{\mathrm{e}}$ siècles, autant lyriques que narratifs, a créé dans l'esprit littéraire une réciprocité topique entre renouveau de l'année et commencement de l'œuvre. Là où la saison chaude servait de cadre à la thématique amoureuse et aventureuse, l'hiver a surtout fait l'objet de personnifications liées la bourgeoisie,

${ }^{1}$ Charles d'Orléans, rondeau 37 (CCCXXXIII), v. 1, dans Ballades et rondeaux, éd. Jean-Claude Mühlethaler, Paris, Le Livre de Poche, 1992, p. 380.

${ }^{2}$ Sur le personnage du vilain et son statut dans la littérature médiévale, on pourra lire Micheline de Combarieu, "Image et représentation du vilain dans les chansons de geste (et dans quelques autres textes médiévaux)», dans Exclus et systèmes d'exclusion dans la littérature et la civilisation médiévales, Aix en Provence, Presses universitaires de Provence, 1978, p. 7-26, http://books.openedition.org/pup/3195 (consulté le 9 septembre 2015); Claire Cabaillot, «La satire du vilain à travers quelques textes du Moyen Âge », Chroniques italiennes, vol. 15, $\mathrm{n}^{\circ} 3, \quad$ 1988, p. 1-27; Isabelle Vedrenne-Fajolles, «Le traitement des stéréotypes dans la Suite du Roman de Merlin : maladresse ou subversion ? De la collision de stéréotypes narratifs avec le type du vilain », Loxias, vol. 17, Littérature à stéréotypes, 2007, http://revel.unice.fr/loxias/index.html?id=1742 et Marie-Thérèse Lorcin, « Du vilain au paysan sur la scène littéraire du XIII ${ }^{\mathrm{e}}$ siècle », Médiévales, vol. 61, 2011, http://medievales.revues.org/6551, (consulté le 9 septembre 2015).

${ }^{3}$ Charles d'Orléans, rondeau 37, dans Ballades et rondeaux, éd. cit., v. 2. 
détachées des canons courtois et aristocratiques. Dans un court Debat de l'Iver et de l'Esté daté de la fin du $\mathrm{Xv}^{\mathrm{e}}$ siècle, le personnage d'Iver se montre essentiellement préoccupé de nourriture lorsqu'il s'adresse à Esté : « Tu as tes beaux potaiges de mes chairs de saison, / Des bons porcz que je tue et metz en salaison ; / Il n'est nul en ce monde qui n'en ait quelque bon $^{4} \gg$. Le mauvais temps symbolisant aisément le mauvais tempérament, l'hiver incarne aussi pour les auteurs l'intrus qu'il faut «banir en essil ${ }^{5} »$. Est-il alors une saison anti-littéraire, renvoyant à ce qui ne pourrait faire l'objet d'une expression artistique ?

Si une «mode » littéraire de la saison obscure naît vers la fin du Moyen Âge, celle-ci constitue selon les critiques une réaction à la tradition précédente: l'apparition des ouvertures automnales et hivernales serait liée à ce que Huizinga a appelé «l'automne du Moyen Âge ${ }^{6} »$, une «tristesse du déjà dit $^{7} »$ qui constitue à cette époque la disposition créative des poètes. La peinture du froid exprime alors un volontaire décalage avec la tradition littéraire, elle fonctionne essentiellement sur un plan intertextuel. On pourrait donc être tenté de

\footnotetext{
${ }^{4}$ Le Debat de l'Iver et de l'Esté, dans Recueil de poesies françoises des XV et XVI $I^{e}$ siècles, morales, facétieuses, historiques, éd. Anatole de Montaiglon, Paris, Jannet, 1857, t. VI, p. 190-195, cit. p. 194. L'éditeur mentionne l'existence de « trois éditions gothiques » de cette œuvre, qu'il ne faut pas confondre avec la pièce contenue dans le manuscrit de Londres, British Library, Harley 2253, fol. 51 et sq., intitulée De l'Yver et de l'Esté.

${ }^{5}$ Charles d'Orléans, rondeau 37, dans Ballades et rondeaux, éd. cit., p. 380. L'hiver partage cette fonction avec le vilain : selon Marie-Thérèse Lorcin ( Du vilain au paysan... », art. cit.), le vilain sert de bouc-émissaire dans cette littérature. Claire Cabaillot indique également que la représentation littéraire du vilain répond au besoin de la société de renforcer "ses défenses en instituant symboliquement un agresseur, un intrus qu'il faut chasser hors de la place » («La satire du vilain à travers quelques textes du Moyen Âge », art. cit., p. 27).

6 Johan Huizinga, L'Automne du Moyen Âge, trad. Julia Bastin, Paris, Payot, 1975. Rappelons que la traduction française de cet ouvrage avait d'abord été intitulée « Le déclin du Moyen Âge ».

${ }^{7}$ Jacqueline Cerquiglini-Toulet, La Couleur de la mélancolie. La fréquentation des livres au XIV siècle 1300-1415, Paris, Hatier, 1993, p. 57-88.
} 
penser que le motif hivernal correspond à l'investissement poétique tardif d'un élément conçu à l'origine comme non littéraire.

En réalité, l'hiver est déjà très présent dans la poésie des troubadours. Tout au long du Moyen Âge, il entre notamment dans la construction de deux topoï littéraires. Le premier consiste en un système opposant hiver et reverdie dans le contexte de la production courtoise (lyrique, puis narrative). Le second relève plutôt de la caractérisation littéraire : il met en relation l'hiver et la pauvreté, la saison étant décrite à travers les signes de la pauvreté et inversement. Nous souhaiterions étudier successivement ces deux lieux communs, à partir de textes qui relèvent de la littérature courtoise et de la «poésie personnelle» du XIII ${ }^{\mathrm{e}}$ siècle $^{8}$. Ces deux constructions topiques se retrouvent dans les textes de Christine de Pizan, où elles permettent à la fois un traitement traditionnel du motif et une construction argumentative complexe: on se penchera donc ensuite sur les effets rhétoriques qui naissent de leur présence simultanée, en analysant en particulier un passage dit «autobiographique » du Livre de l'advision Cristine.

\section{Hiver, désamour et vieillesse}

Présents aux origines du lyrisme roman, l'ouverture printanière et le motif de la reverdie ont rendu topique la corrélation entre l'amour et la saison chaude. Le grand chant courtois des troubadours puis des trouvères établit ainsi une correspondance entre reverdie, chant et

\footnotetext{
${ }^{8}$ Sur cette dénomination de poésie personnelle, voir Michel Zink, La Subjectivité littéraire. Autour du siècle de saint Louis, Paris, PUF, 1985, en particulier p. 47-74, «De la poésie lyrique à la poésie personnelle : l'idéal de l'amour et l'anecdote du moi ».
} 
commencement du texte. Le chant représentant l'expression amoureuse du sujet lyrique, la canso prend pour cadre cohérent la fin de l'hiver et le début de l'été où retentissent le chant des oiseaux et, par imitation, celui du poète ${ }^{9}$. L'hiver est caractérisé à l'inverse par le silence des oiseaux, et correspond souvent dans la lyrique courtoise à une sorte de suspension de l'expression amoureuse ${ }^{10}$. Mais il permet également d'exprimer le paradoxe amoureux, le décalage entre un état naturel de l'amour et son état présent. Comme l'écrit Michel Stanesco, «le début hivernal est un topos bien connu à l'époque de Raimbaut [d'Orange]. Ce procédé sert depuis Marcabru et Cercamon à introduire l'opposition entre le paysage extérieur désolant de la mauvaise saison et la nécessité intérieure du chant amoureux; variante inversée de l'introduction

\footnotetext{
${ }^{9}$ On pourrait citer entre mille, à titre d'exemple, Jaufré Rudel : «Quan lo rius de la fontana / S'esclarzis, si cum far sol, / E par la flors aiglentina, / E'1 rossinholetz el ram / Volf e refranh ez aplana / Son dous chantar et afina, / Dreitz es qu'ieu lo mieu refranha»(Les Chansons de Jaufré Rudel, éd. et trad. Alfred Janroy, Paris, Champion, 1925, «Quand lo rius de la fontana », v. 1-7, p. 3-4). «Quand l'eau de la source court plus claire, comme cela arrive [au printemps], et que paraît la fleur de l'églantier, et que le rossignol, sur la branche, répète, module, adoucit et embellit sa douce chanson, il est bien juste que je module la mienne » (ibid., p. 3-4). Cette équivalence sera ensuite reprise par les trouvères, par exemple Colin Muset: «Mult m'anue d'iver ke tant ait dureit, / Ke je ne voi roisignor en bruel rameit, / Et des ke je voi lou tens renouveleit, / Se me covient ke je soie en cest esteit / Plux mignos et envoixiés ke n'aie esteit» (Les Chansons de Colin Muset. Textes et mélodies, éd. Christopher Callahan et Samuel N. Rosenberg, Paris, Champion, 2005, chanson 10 RS 428, v. 1.1-1.5, p. 157).

${ }^{10}$ On pourrait penser là encore à Jaufré Rudel et sa très célèbre strophe : «Lanquan li jorn son lonc en may / M'es belhs dous chans d'auzelhs de lonh, / E quan mi suy partitz de lay / Remembra'm d'un' amor de lonh : / Vau de talan embroncx e clis / Si que chans ni flors d'albespis / No'm platz plus que l'yverns gelatz» (Les Chansons de Jaufré Rudel, éd. cit., "Lanquan li jorn son lonc en may », v. 1-7, p. 12-13). «Quand les jours sont longs, en mai, il me plaît, le chant des oiseaux, lointain ; et quand je suis parti de là (j'ai cessé de l'écouter), il me souvient d'un amour lointain: je vais alors pensif, morne, tête baissée, et alors ni chant [d'oiseaux], ni fleur d'aubépine ne me plaisent plus que l'hiver glacé » (ibid., p. 12-13).
} 
printanière habituelle, il ne se prolonge jamais au-delà de la première cobla $^{11} \gg$. On rencontre ce type d'ouverture chez Gace Brulé :

Quant flors et glaiz et verdure s'esloingne,

Que cil oisel n'osent un mot soner,

Por la froidour chacuns doute et resoingne

Jusqu'au beau temps que il suelent chanter,

Lors chanterai, que ne puis oblier

La bone amour dont Dex joie me doigne,

Car de li sont et viennent mi penser ${ }^{12}$.

L'hiver construit un paysage à contre-courant du modèle topique le plus répandu, comme le montre au second vers l'usage du démonstratif de notoriété dans «cil oisel ».

Si Michel Zink remarque que

l'usage de commencer toute chanson par une évocation de la nature printanière $[. .$.$] passe de$ mode et est raillé au XIII siècle, parce que, comme l'expliqueront abondamment les trouvères, le véritable amoureux aime en toute saison, et non pas seulement au printemps ${ }^{13}$,

c'est bien cette équivalence symbolique entre reverdie et amour qui se transmettra dans la littérature narrative courtoise, sans doute surtout à travers la réception du Roman de la rose. Le texte composé par Guillaume de Lorris correspond à la mise en roman de la strophe printanière du grand chant courtois ${ }^{14}$; compte tenu de son succès et de son retentissement, il pourrait être à l'origine d'un glissement par lequel cette strophe, « de décor et d'entrée en matière qu'elle était, est

\footnotetext{
${ }^{11}$ Michel Stanesco, «La fleur inverse et la "belle folie" de Raimbaut d'Orange », Cahiers de civilisation médiévale, vol. 40, 1997, p. 233-252, cit. p. 246.

${ }^{12}$ Gace Brulé, «Quant flors et glaiz et verdure s'esloingne », v. 1-7, dans Poèmes d'amour des XII et XIII siècles, éd. et trad. Emmanuèle Baumgartner et Françoise Ferrand, Paris, Union Générale d'Éditions, 1983, p. 50.

${ }^{13}$ Michel Zink, Littérature française du Moyen Âge, Paris, PUF, 1992, p. 110.

${ }^{14}$ Voir par exemple à ce sujet Paul Zumthor, Langue, texte, énigme, Paris, Éditions du Seuil, 1975, p. 249-264 : «Récit et anti-récit : le Roman de la rose ».
} 
devenue, par le jeu du symbole, l'expression même de l'amour ${ }^{15}{ }$. Le début du roman, qui deviendra le modèle de toute narration courtoise, pose très clairement cette équivalence :
Avis m'iere qu'il estoit mais il a ja bien .v. anz ou mais, qu'en may estoie, ce sonjoie, el tens enmoreus, plain de joie, el tens ou toute rien s'esgaie, que l'en ne voit buisson ne haie qui en may parer ne se veille et covrir de novele fuelle. $\mathrm{Li}$ bois recuevrent lor verdure, qui sunt sec tant come yver dure ${ }^{16}$.

Le Roman de la rose ajoute cependant une condition nécessaire à cette équivalence: la jeunesse. Le narrateur précise d'emblée que l'action se déroule «El vintieme an de [s]on aage, / el point qu'Amors prent le paage / des jones genz ${ }^{17} \gg$. Même si la représentation de l'hiver sous les traits d'un vieillard est très ancienne, Le Roman de la rose participe à en faire le symbole d'un repoussoir courtois : l'exclusion du public vieilli est confirmée par la présence d'une représentation de Vieillesse sur le mur extérieur du verger, parmi les vices destinés à rester hors du domaine amoureux ${ }^{18}$.

Cette triple équivalence anti-courtoise entre l'hiver, le désamour et la vieillesse se retrouve tout au long du Moyen Âge. On la rencontre dans le Debat de l'Iver et de l'Esté où le second dit au premier :

\footnotetext{
${ }^{15}$ Michel Zink, Roman rose et rose rouge : le Roman de la rose ou de Guillaume de Dole de Jean Renart, Paris, Nizet, 1979, p. 75.

${ }^{16}$ Guillaume de Lorris et Jean de Meun, Le Roman de la rose, éd. Lecoy, Paris, Champion, 1965-1970, 3 t., t. I, 1970 [1965], v. 47-54. Le narrateur précise d'ailleurs juste après : " Mout a dur cuer qui en may n'aime, / quant il ot chanter sus la raime / as oisiaus les douz chans piteus ». ibid., v. 81-83.

17 Ibid., v. 21-24. La mention de l'âge sera ensuite relayée dans le texte par l'expression «jeunes gens» («lors estuet joines genz entendre / a estre gais et amoreus / por le tens bel et doucereus » : ibid., v. 78-80).

${ }^{18}$ Ibid., v. 339-404.
} 
Yver, tu n'as desir que de ta pance emplir ;

Mieux vault en ung vergier dessus l'herbe

gesir,

En acollant [s'amye], et baisier a loisir,

Que le feu où te chauffes, qui ne fait

qu'envieillir ${ }^{19}$.

L'équivalence entre reverdie, amour et jeunesse était quant à elle si complète que les dits amoureux de la fin du Moyen Âge en ont souvent inversé les termes : la jeunesse du narrateur, première mentionnée dans le texte, appelle nécessairement la thématique amoureuse, qui engendre à son tour l'apparition du motif printanier ${ }^{20}$. C'est donc un fait acquis à la fin du Moyen Âge : l'hiver est le temps du vide et du silence, du désamour souvent lié au vieil âge. Toute parole y prenant place est paradoxale, soit parce qu'elle entre en contradiction avec le cadre qui l'accueille, soit parce qu'elle s'oppose à la topique littéraire dominante. L'ouverture du Lais de Villon en témoigne :

En ce temps que j'ay dit devant, Sur le Noël, morte saison,

Que les loups se vivent de vent

Et qu'on se tient en sa maison,

Pour le frimas, pres du tyson, me vint ung vouloir de briser

\footnotetext{
${ }^{19}$ Le Debat de l'Iver et de l'Esté, éd. cit., p. 193.

${ }^{20}$ Les auteurs semblent vouloir insister d'abord sur le très jeune âge de l'amant narrateur, comme pour reculer sans cesse la limite «basse » de l'amour. On pourrait penser par exemple à L'Espinette amoureuse de Jean Froissart, qui s'étend longuement sur les relations entre le jeune âge et l'amour avant de raconter la première histoire d'amour véritable du narrateur, qui prendra pour décor le «joli mois de may »: Jean Froissart, L'Espinette amoureuse. Seconde édition entièrement revue, éd. Anthime Fourrier, Paris, Klincksieck, 1972, v. 351. Dans le même registre, Le Livre du duc des vrais amans de Christine de Pizan s'ouvre sur ces vers du narrateur: "Joenne et moult enfant estoye/ Quant ja grant peine mettoye / A amoureux devenir » (Christine de Pizan, Le Livre du Duc des vrais amans, éd. Dominique Demartini et Didier Lechat, Paris, Champion, 2013, v. 41$43)$; le récit rapportera ensuite la première rencontre des amants dans un décor printanier.
} 
La tres amoureuse prison

Qui faisoit mon cueur debriser ${ }^{21}$.

L'hiver annonce un départ, la fin de l'amour dont l'été marquait le commencement.

La mention du frimas et du tison, dans laquelle se devine un répertoire d'images propre à la saison froide, fournit les accessoires d'un «temps mort» où la narrativité humaine se suspend: le retrait dans la maison répond au vent d'hiver, symbole de la vacuité et de la vanité du monde. La caractérisation de l'hiver prend appui sur un ressenti humain : elle est éternellement affaire de faim, de fatigue et de froid.

« En yver, du feu, du feu $!^{22} »:$ hiver et pauvreté

L'hiver n'est la plupart du temps décrit qu'à travers un climat et ses conséquences sociales. Le froid qui le caractérise oppose ceux qui peuvent s'en protéger et ceux qui ne le peuvent pas. La présentation de cette saison dans le Debat de l'Iver et de l'Esté convoque donc nécessairement des classes sociales opposées.

Esté, j'ay nom Yver, qui, par maintes contrées

Envoye de mes biens, pluyes, neiges et gelées ;

Partout là où je suis sont froidures trouvées, Je fais aux enrichis vestir robes fourrées ${ }^{23}$

Yver, en celuy temps, qui n'a rien que vestir, Quand il vente ou il neige, pleut ou fait grant gresil,

${ }^{21}$ Le Lais François Villon, v. 10-16, dans François Villon, Euvres complètes, éd. Jacqueline Cerquiglini-Toulet, Paris, Gallimard, 2014, p. 3. Sur cette strophe et plus généralement sur les saisons chez Villon, on pourra lire Barbara N. SargentBaur, "L'hiver et l'été dans l'œuvre de François Villon », "Pour acquerir honneur et pris". Mélanges de Moyen Français offerts à Giuseppe Di Stefano, dir. Maria Colombo Timelli et Claudio Galderisi, Montréal, CERES, 2004, p. 339-344.

${ }^{22}$ Charles d'Orléans, rondeau 283 (CCCXLVIII), v. 1, dans Ballades et rondeaux, éd. cit., p. 634.

${ }^{23}$ Le Debat de l'Iver et de l'Esté, éd. cit., p. 192. 
Tout le menu commun, vit en grant desplaisir ;

Povres membres de Dieu si ont bien a souffrir ${ }^{24}$.

Lorsqu'il est dépeint sous un jour positif, l'hiver est perçu à travers l'univers bourgeois :

Esté, en ce bon temps j'ay de grans assemblées ;

J'ay bourgeois et marchans a grans robes fourrées,

Houseaulx et bons manteaux, et les chesnes dorées ;

Pour moy font beau grant feu et fumer cheminées ${ }^{25}$.

Mais la majorité des caractérisants de l'hiver sont ceux des miséreux. $\mathrm{Au}$ feu et aux vêtements du confort bourgeois s'oppose Povreté, telle qu'elle est représentée sur le mur extérieur du verger dans le Roman de la rose, rejetée comme Vieillesse hors du monde amoureux et courtois :

tant seüst bien sa robe vendre, qu'ele estoit nue come vers.

Se li tens fust un poi divers ${ }^{26}$, je cuit qu'ele acorast de froit, qu'el n'avoit c'un viez sac estroit, tot plain de mauvés paletiaus :

c'estoit sa cote et ses mantiaus ; el n'avoit plus que afubler, grant loisir avoit de trembler ${ }^{27}$.

Le pauvre, par opposition aux bourgeois et aux nobles, est celui qui souffre du froid et de la faim, dans un contexte saisonnier où la nourriture vient à manquer. Cette corrélation s'observe dans le portrait que Le Livre de la mutacion de fortune de Christine de Pizan dresse de Pauvreté en s'inspirant du Roman de la rose. La faim y figure parmi d'autres traits caractéristiques liés ou non au contexte hivernal, comme le mauvais feu ou le manque de sommeil.

\footnotetext{
${ }^{24}$ Ibid., p. 195.

${ }^{25}$ Ibid., p. 194.

${ }^{26}$ Armand Strubel, dans son édition du texte, choisit d'éditer : « Se li tens fust i. pou d'yvers ». Guillaume de Lorris et Jean de Meun, Le Roman de la rose, éd. Armand Strubel, Paris, Le Livre de poche, 1992, v. 446, p. 62

${ }^{27}$ Guillaume de Lorris, Le Roman de la rose, éd. Felix Lecoy, éd. cit., v. 442-450.
} 
Toute semble estre alangouree

De povreté, de froit, de fain,

Et de trembler avoit grant fain.

Ne sçay comment froit ne la tue

En yver, car elle est vestue

De linges draps trop povrement [...].

Si croy qu'elle ait petit repos,

Car, ou le preigne ou non en gré,

Un pou d'estrain soubs un degré

A seulement pour soy coucher,

Ne autre lit paré ne chier,

Povre maison et enfumee,

Ou pou de feu fait grant fumee,

Ou il n'a se souffreté non ${ }^{28}$.

Ces représentations de l'indigence, dont l'archétype est en réalité très ancien, oscillent entre un type d'écriture « réaliste ${ }^{29} »$ (comme le portrait de Pauvreté dans la Mutacion de fortune) et des choix plus symboliques, qui opèrent à travers un prisme littéraire. Les deux cas se rencontrent chez Rutebeuf. Dans La Complainte Rutebuef, les malheurs du narrateur sont énumérés dans un cadre réaliste :

Ce je m'esmai, je n'en puis mais,

Car je n'ai douzainne ne fais,

En ma maison,

De buche por ceste saison. [...]

Mes hostes wet l'argent avoir

De son hosteil,

Et j'en ai presque tout ostei,

Et si me sunt nu li costei

${ }^{28}$ Christine de Pizan, Le Livre de la mutacion de Fortune, éd. Suzanne Solente, Paris, Picard, 1959-1966, 4 t., t. I, 1959, v. 2590-2609.

${ }^{29}$ Par le terme de « réalisme », la critique se réfère souvent à la production textuelle du XIII siècle constituée par le corpus des romans réalistes et la «poésie personnelle ». Pour les romans, voir par exemple Michel Zink, Littérature française du Moyen Âge, op. cit., «Les romans dits "réalistes" », p. 158-166. Michel Zink note à propos de la question du réalisme chez Rutebeuf : "Cette poésie des choses de la vie n'a nullement pour préalable une exigence de sincérité, contrairement à la poésie courtoise [...] ; elle vise seulement à une dramatisation concrète du moi. C'est une poésie de la réalité particulière et reconnaissable, mais travestie, comme est particulier et travesti le moi qui l'expose et qui s'expose ». (Michel Zink, La Subjectivité littéraire, op. cit., p. 63). 
Contre l'iver [...].

Mei gage sunt tuit engaigié

Et d'enchiez moi desmenagiei,

Car g'ai geü

Trois mois, que nelui n'ai veü ${ }^{30}$.

Ailleurs, c'est cependant à la littérature que la complainte fait appel, par une allusion à $\mathrm{Job}^{31}$, figure d'identification récurrente pour le pauvre et modèle fondateur du geste d'écriture énumérateur. Le prisme littéraire peut également se manifester dans une relecture ironique de la topique courtoise, comme c'est le cas dans La Griesche d'hiver:

Je ne dor que le premier soume. [...]

Ausi sui con l'oziere franche

Ou com li oiziaux seur la branche :

En estei chante,

En yver pleure et me gaimente,

Et me despoille ausi com l'ante

Au premier giel ${ }^{32}$.

Parmi les maux qui frappent le pauvre (le mauvais sommeil, les huissiers, les enfants à charge ${ }^{33}$, l'isolement social), une grande part est irréductiblement liée au contexte hivernal : la faim, le froid, le manque de vêtement et de bois, la cheminée qui fume d'un mauvais feu, la maladie enfin ${ }^{34}$. Si l'hiver se définit nécessairement par ses conséquences sociales, la pauvreté est représentée durant la saison qui en exacerbe le ressenti.

30 La Complainte Rutebuef de son oeul, v. 68-95, dans Rutebeuf, Euvres complètes, éd. Michel Zink, Paris, Le Livre de poche, 1989-1990, p. 322.

${ }^{31}$ Ibid., v. 20-22, p. 320 : «Diex m'a fait compaignon a Job : / Il m'a tolu a un sol cop / Quanque j'avoie ».

${ }^{32}$ Li diz de la griesche d'yver, v. 28-39, dans Rutebeuf, Euvres complètes, éd. cit., p. 196-198.

33 La Complainte Rutebuef de son oeul, v. 51-64, dans Rutebeuf, CEuvres complètes, éd. cit., p. 320 : «Or a d'enfant geü ma fame ; [...] / Or wet de l'argent ma norrice, / Qui m'en destraint et m'en pelice / Por l'enfant paistre, / Ou il revanrra braire en l'aitre. / Cil sire Diex qui le fit naitre / Li doint chevance / Et li envoit sa soutenance, / Et me doint ancor alijance / Qu'aidier li puisse ».

${ }^{34}$ Ibid., v. 20-38 et v. 94-106, p. 318-324. 


\section{La jeunesse hivernale de Christine de Pizan}

Comme en témoigne Rutebeuf, la rencontre des lieux communs liant l'hiver au désamour et à la pauvreté permet des constructions rhétoriques dont l'interprétation dépasse l'explicite du texte : dans $L a$ Griesche d'hiver, la réécriture de la reverdie sur un mode hivernal, qui s'appuie sur une identification topique du sujet lyrique à l'oiseau, correspond à un procédé d'ironie ${ }^{35}$. Ailleurs, ces constructions rhétoriques peuvent acquérir une valeur argumentative fonctionnant sur un plan presque subliminal : c'est le cas dans la troisième partie du Livre de l'advision Cristine, composé par Christine de Pizan en $1405^{36}$. Dans cette partie qu'on a souvent qualifiée d'autobiographique ${ }^{37}$, Christine rencontre Philosophie dans les combles de l'université de Paris et lui raconte les malheurs de son existence. Après avoir retracé le temps heureux de son enfance et de ses dix années de mariage, Christine évoque les morts successives du roi protecteur de son père, de son père puis de son mari. Demeurée seule à Paris avec sa mère, ses

\footnotetext{
${ }^{35}$ Comme l'écrit Michel Zink, la poésie de Rutebeuf «prend le contrepied du grand chant courtois en substituant à la généralisation l'anecdote et à l'idéalisation la satire » : on reconnaît dans cette réécriture ironique de la reverdie un tel processus de dés-idéalisation. (Michel Zink, La Subjectivité littéraire, op. cit., p. 62).

${ }^{36}$ Christine de Pizan, Le Livre de l'advision de Cristine, éd. Christine Reno et Liliane Dulac, Paris, Champion, 2001.

${ }^{37}$ Parmi les nombreuses publications sur L'Advision Cristine qui ont fait usage de ce terme, on pourra consulter, classés par date de publication, Rosalind BrownGrant, «L'Avision-Christine : autobiographical narrative or mirror for the princes ? », dans Politics, gender and genre: the political thought of Christine de Pizan, dir. Margaret Brabant, Boulder, Westview Press, 1992, p. 95-111 ; Mary L. Skemp, «Autobiography as authority in Lavision-Christine », Le Moyen Français, vol. 35, 1996, p. 17-31; Jean-Philippe Beaulieu, «L'Avision Christine ou la tentation autobiographique », Littératures, vol. 18, 1998, p. 15-30 et Anne Paupert, "La narracion de mes aventures": des premiers poèmes à L'Advision, l'élaboration d'une écriture autobiographique dans l'œuvre de Christine de Pizan », dans $A u$ champ des escriptures. III Colloque international sur Christine de Pizan, Lausanne, 18-22 juillet 1998, dir. Eric Hicks, Diego Gonzalez et Philippe Simon, Paris, Champion, 2000, p. 51-71.
} 
trois enfants et une nièce à sa charge, Christine rencontre de très nombreux problèmes financiers :

O doulce maistresse, quantes larmes, souspirs, plaints, lamentacions et grieves pointures cuides tu, quant je estoie seulete en mon retrait, que je eusse et gectasse en ce tendis, ou quant a mon foyer veoie environ moy mes petis enfans et povres parens et consideroie le temps passé et les infortunes presentes dont les floz si bas m'affondoient, et remedier n'y pouoie ! [...] Et avec ce cuides tu point, chiere maistresse, que grevast a mon cuer la charge de la paour que on s'apperceust de mes affaires et le soussi que a l'estat n'apparust a ceulz dehors ne aux voisins le decheement de ce maleureux estat venu de mes predecesseurs, non pas de moy; lequel ignorance tant amer me faisoit que mieulx eusse choisi mourir qu'en decheoir ? [...] Si te prometz que a mes semblans et abis pou apparoit entre gens le faissel de mes anuys. Ains soubz mantel fourré de gris et soubz surcot d'escarlate, non pas souvent renouvellé mais bien gardé, avoie espesses fois de grans friçons, et en biau lit et bien ordonné de males nuis. Mais le repast estoit sobre, comme il affiere a femme vesve ; et toutesfois vivre convient. Et Dieu scet comment mon cuer tourmenté estoit quant execucions par sergens sur moy estoient faictes et mes chosetes m'estoient levees ! Le dommaige grant m'estoit, mais plus craignoit la honte. Mais quant il convenoit que je feisse aucun emprumpt ou que soit pour eschever plus grant inconvenient, biaux sire Dieux, comment honteusement, a face rougie - tant fust la personne de mon amistié - le requeroie! Et encore au jour d'ui garie ne suis d'icelle maladie, dont tant ne me greveroit, comme il me semble, quant faire le m'esteut, ung axcés de fievre.

Ha! quant il me souvient comment tant de fois ay musé la matinee a ce palais en yver, mourant de froit, espiant ceulz de mon conseil pour ramentevoir et solliciter ma besoingne, ou mainte fois y ouoye a mes journees de diverses conclusions qui suer des yeulx me faisoient et maintes estranges responses! Ensurquetout me grevoit la mise de laquelle mal estoie aisiee.

Et a l'example de Jhesucrist qui voult estre tourmenté en toutes les parties de son corps pour nous instruire a pascience, voult Fortune que mon cuer fust tourmenté de 
toutes manieres de dures et desplaisans pensees diversement. Quel plus grant mal et desplaisir puet sourdre a l'innocent, ne plus grant cause d'impacience, que de soi ouir diffamer sans cause, comme il appert par les recors de Boece en son Livre de consolacion? Ne fut il pas dit de moy par toute la ville que j'amoie par amours ? Voire, mais yci fait trop a noter que il soit voir que tout ce faisoit Fortune par ses batemens divers. Car, comme telz renommees viennent et sourdent communement, et souvent a tort, par grant acointance et frequentacions les personnes ensemble ou par conjectures et couleurs voirs semblables mais je te jure m'ame que ycelui ne me congnoissoit ne ne sçavoit qui j'estoie, ne oncques ne fu homme ne creature nee qui en publique n'a privé me veist soubz toit n'en lieu n'en place ou il fust, car mon chemin ne s'i adonnoit ne n'y avoie que faire, et de ce me soit Dieux tesmoing que je dis voir $^{38}$.

Ce passage présente deux caractéristiques intéressantes. D'une part, la thématique des problèmes financiers est assez brusquement remplacée par celle de la rumeur amoureuse, sans que le texte n'annonce clairement un changement de sujet; la suite du chapitre retournera d'ailleurs au thème financier. D'autre part, si cette transition paraît abrupte sur le plan thématique, elle est très travaillée sur le plan stylistique. La conjonction de coordination «Et» qui ouvre le dernier paragraphe crée un lien entre ce qui précède et ce qui suit ; l'allusion aux douleurs du Christ et à celles de Boèce semble indiquer un renchérissement dans le récit, propre à ces écritures énumératrices dont Le Livre de Job constitue le modèle fondateur. Quelle est alors la nature de ce lien suggéré entre problèmes d'argent et rumeur amoureuse ?

Les difficultés financières de Christine ne furent pas de courte durée, comme elle l'indique elle-même : «Et ne cuides mie que m'ait duré ung an ou deux ; mais l'espace de plus de .XIIII. ans ${ }^{39} »$. Or, dans

\footnotetext{
38 Christine de Pizan, Le Livre de l'advision Cristine, op. cit., partie III, chapitre 6, 1. 92-148, p. 102-104.

${ }^{39}$ Ibid., 1. 85-86, p. 102.
} 
le chapitre six, tous les indices temporels placent la scène en hiver : «Ha!», s'exclame Christine, « quant il me souvient comment tant de fois ay musé la matinee a ce palais en yver, mourant de froit ${ }^{40} »$. On retrouve les caractérisants topiques de l'hiver, comme la famille réunie au foyer («quant a mon foyer veoie environ moy mes petis enfans et povres parens $\left.{ }^{41} »\right)$, dans un portrait qui conjoint le motif des enfants à charge et l'imagerie de la famille rassemblée autour du feu, telle qu'on la rencontre dans les enluminures des livres d'heures. Y sont également mentionnés les habits d'hiver, associés à la maladie («Ains soubz mantel fourré de gris et soubz surcot d'escarlate, non pas souvent renouvellé mais bien gardé, avoie espesses fois de grans friçons $\left.{ }^{42} »\right)$. Le motif de la maladie fait l'objet de plusieurs développements: métaphorique, lorsque Christine compare la difficulté à emprunter de l'argent à des accès de fièvre, réaliste à propos du manteau fourré ${ }^{43}$. D'autres caractérisants présents dans le passage sont ceux qui définissent la pauvreté sans référence à un contexte hivernal : ainsi de la mention des huissiers ${ }^{44}$ ou du mauvais sommeil ${ }^{45}$.

La tonalité hivernale qui traverse ce passage est donc cohérente avec la peinture des problèmes financiers de Christine. Propre à attirer la compassion du lecteur, cette écriture à visée argumentative fait usage de micro-descriptions, extrêmement économiques sur le plan textuel. Elle donne à voir des scènes fugitives proches tout à la fois de ce qui

\footnotetext{
${ }^{40}$ Ibid., 1. 126-127, p. 103.

${ }^{41}$ Ibid., 1. 94-95, p. 102.

${ }^{42}$ Ibid., 1. 113-115, p. 103.

43 On pourrait ajouter à ces deux occurrences une troisième qui précède de peu notre passage et qui compare la narratrice au personnage de Job : «Affin que je parvenisse au point ou Fortune me conduisoit en ce temps ou comble de mes adversitez fortune[es], me sourdi comme a Job longue maladie ». ibid., 1. 65-67, p. 101.

${ }^{44}$ Ibid., 1. 117-120, p. 103.

45 « et en biau lit et bien ordonné de males nuis ». ibid., 1. 115-116, p. 103.
} 
constituera plus tard la peinture de genre et de ce que Barthes appelait des « biographèmes ${ }^{46}$ »: une famille rassemblée autour de la mère, un repas de veuve, des huissiers se présentant au domicile à la vue des voisins, des matinées d'hiver au palais de justice. Ces scènes de la vie quotidienne font sens pour le lecteur parce qu'elles s'appuient sur une topique largement répandue et reconnaissable ; elles se complètent les unes les autres jusqu'à construire une représentation traditionnelle de la pauvreté hivernale.

\section{La jeunesse à rebours}

L'ancrage hivernal du chapitre six prend un autre sens dans la construction du passage, qui évoque après les soucis financiers la rumeur d'une liaison amoureuse ayant couru sur l'auteur. Lors de la rédaction de l'Advision Cristine, soit une quinzaine d'années après les événements racontés, cette rumeur possède encore pour Christine une dimension polémique ; il serait sinon peu probable que la narratrice ait évoqué d'elle-même le sujet. Or la construction rhétorique du passage permet à elle seule d'invalider les allégations dont Christine a fait l'objet. L'évocation des problèmes financiers constitue la preuve judiciaire que la rumeur est sans fondement.

La période hivernale est en elle-même un cadre où l'amour ne peut topiquement pas prendre place, mais la structure énumérative du texte réactive également un autre lien possible entre l'hiver et la pauvreté : celui de la besogne, de l'activité constante découlant des

\footnotetext{
${ }^{46}$ Roland Barthes, Sade, Fourier, Loyola, dans Euvres complètes, éd. Éric Marty, Paris, Le Seuil, t. III, 1995, p. 1045 : «Si j'étais écrivain et mort, comme j'aimerais que ma vie se réduisît, par les soins d'un biographe amical et désinvolte, à quelques détails, à quelques goûts, à quelques inflexions, disons des "biographèmes" dont la distinction et la mobilité pourraient voyager hors de tout destin et venir toucher, à la manière des atomes épicuriens, quelque corps futur, promis à la même dispersion ; une vie "trouée", en somme, comme Proust a su écrire la sienne dans son œuvre ».
} 
problèmes d'argent. Rappelons que Le Roman de la rose soulignait la nécessité, pour accéder au monde de l'amour, d'une liberté d'occupation représentée par Oiseuse, la portière du verger. Les déambulations financières de Christine servent donc à celle-ci d'alibi à la fois géographique (elles confirment que «[s]on chemin ne $\mathrm{s}^{\prime}[\ldots]$ adonnoit $\gg$ vers les cercles où évoluait son prétendu amant ${ }^{47}$ ) et symbolique (le besogneux ne peut être amoureux). Une dernière valeur de l'hiver est également active dans le chapitre six : après la description d'une jeunesse heureuse, l'omniprésence de l'hiver dans le récit des infortunes de Christine semble transposer sur un plan cosmologique le vieillissement de la narratrice.

Occupation et vieillissement sont reconvoqués au début du chapitre suivant, qui explicite la construction rhétorique de notre passage : «Entens tu, doulce maistresse, en quelz doulz deduis ay passé la jeunesce de ma vesveté ! Avoie cause qui trop druerie me feist entendre aux foles amours $?^{48} »$. La contradiction entre la réalité décrite et le contexte courtois (convoqué peut-être ironiquement dans l'emploi du mot deduit, nom du propriétaire du verger dans le Roman de la rose) est entérinée par cette antiphrase qui clôt définitivement le sujet.

\section{Le monde paradoxal}

Dans L'Advision Cristine, la topique hivernale n'a pas une valeur uniquement argumentative. Elle permet également de décrire un monde apparemment contradictoire. Le paradoxe de l'existence de Christine réside dans un vieillissement prématuré dû à la disparition de son mari, figure double de l'amant et du chef de famille. L'hiver est une manière d'apprivoiser l'expérience de la perte à la fois affective et matérielle, en

\footnotetext{
${ }^{47}$ Christine de Pizan, Le Livre de l'advision Cristine, éd. cit., 1. 146-147, p. 104.

${ }^{48}$ Ibid., partie III, chap. 7, 1. 2-4.
} 
l'englobant dans un processus d'inversion des saisons: le lien symbolique entre saisons et âges de la vie est brisé mais le cycle naturel permettra au parcours linéaire de la vie de connaître une renaissance. À cet hiver tombé sur la jeunesse succèdera dans les chapitres suivants un nouveau printemps : la découverte de l'estude, c'est-à-dire de la lecture, puis de l'écriture ${ }^{49}$. Cette réapparition printanière du bonheur est métaphorisée dans Le Livre du chemin de long estude, qui place son action dans la nuit du 5 octobre 1402 et possède une ouverture à la fois nocturne et automnale. Lorsque la sibylle de Cumes propose à Christine de voyager à travers le monde pour apaiser la douleur du deuil, la narratrice précise : «Touret de nez je mis et guimple, / Pour le vent qui plus grieve a l'ueil/ En octobre que grant souleil $»^{50}$. En suivant la sibylle, Christine se retrouve pourtant presque immédiatement sur une voie printanière :

Ainsi flourie la trouvames

Et verdoyant d'erbe menue

Et tout en l'estat maintenue

Que ou mois de may sont les vers prez

De plusieurs couleurs dyaprez.

Lors m'est droitement souvenu

Que le doulx may fust revenu,

Tant senti attrempé le temps ${ }^{51}$.

Si la perte du chef de famille provoque pour Christine la chute brutale de sa jeunesse dans un hiver prématuré - celui du désamour et de la pauvreté - le réconfort de l'étude représente le retour paradoxal à une première saison de la vie, commencée à «la moitié du chemin de

\footnotetext{
${ }^{49}$ Ibid., partie III, chap. 10 («Dit Crisine comment elle se mist a l'estude ») et 11 ( «Le plaisir que Cristine prenoit a l'estude»).

${ }^{50}$ Christine de Pizan, Le Chemin de Longue Étude, éd. Andrea Tarnowski, Paris, Le Livre de poche, 2000, v. 702-704, p. 128.

${ }^{51}$ Ibid., v. 718-725, p. 130.
} 
[s]on pelerinage » sur terre ${ }^{52}$. Ce second printemps substitue néanmoins la science à l'amour. Dans Le Livre du chemin de long estude, les fleurs sont admirées non plus comme des éléments constitutifs de la topique amoureuse $^{53}$ mais pour leurs vertus curatives :

Et ces flourettes odorans

Par les chemins sont dru semees,

Në il n'est belles fleurs amees,

Roses, violettes ne lis

Ne belles fleurs n'autres delis

Ne chose bonne a medicine,

Prouffitable herbe, flour, racine

Nez poulieul, ysoppe et mante,

$\mathrm{Ne}$ cuidez mie que je mente,

Dont tout le lieu ne soit semé ${ }^{54}$.

L'estude, tout en offrant une reverdie à l'existence, permet aussi d'en évacuer la question amoureuse.

On a beaucoup écrit sur l'obscurité de «La fleur inverse » de Raimbaut d'Orange :

Ar resplan la flors enversa

Pels trencans rancx e pels tertres,

Cals flors? Neus, gels e conglapis

Que cotz e destrenh e trenca,

Don vey morz quils, critz, brays, siscles

En fuelhs, en rams e en giscles ;

${ }^{52}$ Christine de Pizan, Le Livre de l'advision Cristine, éd. cit., partie I, chap. 1, 1. 2, p. 11 .

${ }^{53}$ Les fleurs sont une composante courante du locus amoenus, depuis longtemps lié à l'amour puis qu'on le décrit de la façon suivante «[d]ans le lexique de Papias (environ 1050) : amoena loca dicta : quod amorem praestant, jocunda, viridia », (Ernst Robert Curtius, La Littérature européenne et le Moyen Âge latin, trad. Jean Bréjoux, Paris, PUF, 1956, p. 241, et plus largement p. 240-244 sur le locus amoenus).

${ }^{54}$ Christine de Pizan, Le Chemin de Longue Étude, éd. cit., v. 776-785, p. 132-134. Cette reverdie de l'intellect est aussi symbolisée par la présence de la fontaine de Sapience sur le chemin nommé «Lonc Estude», fontaine inspirée de l'Ovide moralisé qui forme un contrepoint avec la fontaine amoureuse de Narcisse figurant dans Le Roman de la rose. 
Mas mi ten vert e jauzen Joys

Er quan vey secx los dolens croys 55 .

Or cette obscurité rejoint la tension poétique qui habite la topique hivernale : cette possibilité de symboliser l'expérience paradoxale du monde par le sujet littéraire. S'inscrivant dans un cycle, l'hiver provoque une souffrance qui n'annihile pas toute joie; à l'image du destin de Job, il représente souvent la perte nécessaire à l'avènement d'une joie nouvelle ${ }^{56}$. C'est ce gain paradoxal que Philosophie met en évidence dans la vie de Christine :

il n'est mie doubte que, se ton mary t'eust duré jusques a ore, l'estude tant comme tu as n'eusses frequenté, car occupacion de mainage ne le t'eust souffert [...]. Lequel bien d'estude je sçay que tu confesseras que pour tous les biens de Fortune ne vouldroies, quelque pou que y aies fait, ne t'y estre occupee, et que la delectacion qui tant t'en agree ne eusses $^{57}$.

Grâce au cycle des saisons, la symbolique de la reverdie finit par être anticipée dans l'hiver grâce à une perspective chrétienne, où les tribulations de la vie sur Terre préparent les joies du Paradis. Cette opération intellectuelle et poétique de renversement des valeurs

55 Raimbaut d'Orange, «Ar resplan la flors enversa», dans Songs of the troubadours and trouvères : an anthology of poems and melodies, éd. Samuel N. Rosenberg, Margaret Switten, Gérard Le Vot, New York/Londres, Garland, 1998, p. 72-73, cit. p. 72, v. 1.1-1.8. "Quand paraît la fleur inverse / Sur rocs rugueux et sur tertres, / - Est-ce fleur? Non, gel et givre / Qui brûle, torture et tronque ! - / Morts sont cris, bruits, sons qui sifflent / En feuilles, en rains, en ronces. / Mais me tient vert et joyeux Joie, / Quand je vois secs les âcres traîtres » (Pierre Bec, Anthologie des troubadours. Édition bilingue, Paris, Union générale d'édition, 1979, p. 150). Sur l'interprétation de ce poème, voir Michel Stanesco, «La fleur inverse et la "belle folie" de Raimbaut d'Orange », art. cit.

${ }^{56}$ Michel Stanesco le souligne à propos de Raimbaut d'Orange : «Le motif du monde renversé est bien un lieu commun. Mais alors que d'autres troubadours l'utilisent pour des raisons morales [...] Raimbaut d'Orange le récupère en un sens positif ». «La fleur inverse et la "belle folie" de Raimbaut d'Orange », art. cit., p. 252.

57 Christine de Pizan, Le Livre de l'advision Cristine, éd. cit., partie III, chap. 18, 1. 22-30, p. 123. 
mondaines était bien exprimée par Raimbaut d'Orange : «Quar enaissi m'o enverse ${ }^{58} »$. L'hiver constitue la liberté du déshérité : plutôt qu'anti-littéraire, il est, dans ces contextes qui échappent à la norme poétique, un curseur d'inversion du monde par le sujet littéraire. Il permet de saisir une existence au-delà, mais toujours au travers de la topique littéraire, d'exprimer l'espoir d'un bonheur issu du sacrifice et de la mort :

J'ai hiverné dans mon passé

Revienne le soleil de Pâques ${ }^{59}$.

${ }^{58}$ Raimbaut d'Orange, «Ar resplan la flors enversa », éd. cit., p. 73, v. 2.1. Pierre Bec choisit de traduire: "Car le monde ainsi j'inverse» (Anthologie des troubadours. Édition bilingue, éd. cit., p. 151). Jacques Roubaud traduit quant à lui «Ainsi toutes choses j'inverse» (Les Troubadours. Anthologie bilingue, Paris, Seghers, 1971, p. 145).

59 Guillaume Apollinaire, «La Chanson du Mal-Aimé », Alcools, Paris, Gallimard, Paris, 1966, p. 19. 